
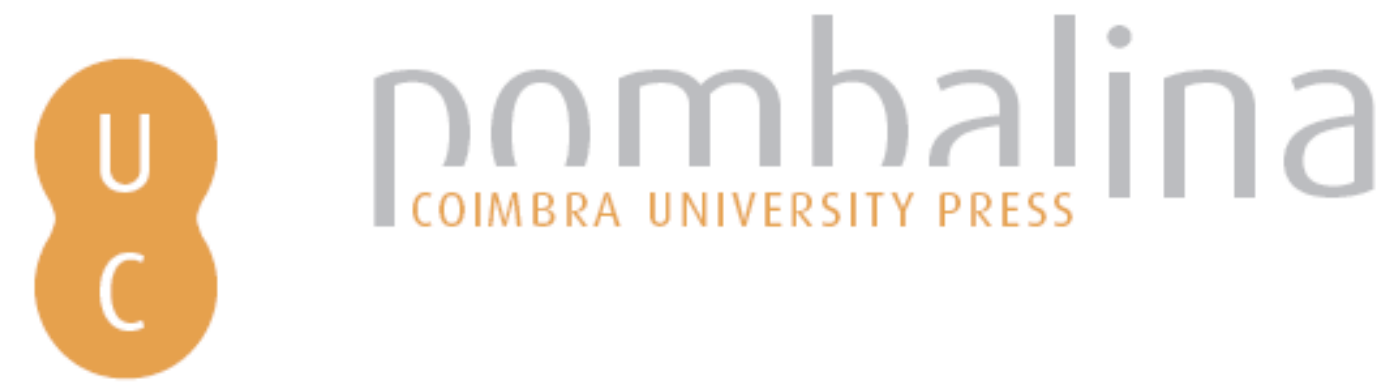

\title{
Economia e interdisciplinaridade: porque é que a economia não pode ser deixada apenas aos economistas?
}

\author{
Autor(es): Neves, Vítor \\ Publicado por: Imprensa da Universidade de Coimbra \\ URL \\ persistente: URI:http://hdl.handle.net/10316.2/40065 \\ DOI: $\quad$ DOl:https://doi.org/10.14195/978-989-26-1239-3_4 \\ Accessed : $\quad$ 26-Apr-2023 16:28:37
}

A navegação consulta e descarregamento dos títulos inseridos nas Bibliotecas Digitais UC Digitalis, UC Pombalina e UC Impactum, pressupõem a aceitação plena e sem reservas dos Termos e Condições de Uso destas Bibliotecas Digitais, disponíveis em https://digitalis.uc.pt/pt-pt/termos.

Conforme exposto nos referidos Termos e Condições de Uso, o descarregamento de títulos de acesso restrito requer uma licença válida de autorização devendo o utilizador aceder ao(s) documento(s) a partir de um endereço de IP da instituição detentora da supramencionada licença.

Ao utilizador é apenas permitido o descarregamento para uso pessoal, pelo que o emprego do(s) título(s) descarregado(s) para outro fim, designadamente comercial, carece de autorização do respetivo autor ou editor da obra.

Na medida em que todas as obras da UC Digitalis se encontram protegidas pelo Código do Direito de Autor e Direitos Conexos e demais legislação aplicável, toda a cópia, parcial ou total, deste documento, nos casos em que é legalmente admitida, deverá conter ou fazer-se acompanhar por este aviso.

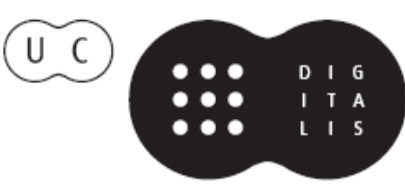




\section{Conrefencicas

INTERDISCIPLINARIDADE E UNIVERSIDADE

ANTÓNIO RAFAELAMARO ÁLVARO GARRIDO JOÃO PAULO AVELÃS NUNES

COORDENAÇÃO 


\section{ECONOMIA E I NTERDISCIPLINARIDADE}

\section{PORQue É QUE A ECONOMIA NÃO PODE SER DEIXAda Apenas aOs ECONOMISTAS?}

Vítor Neves Universidade de Coimbra

\section{RESUMO}

Este é um texto sobre Economia e interdisciplinaridade. Parte da convicção de que pensar a interdisciplinaridade é pensar a natureza da própria disciplinaridade, de que falar de interdisciplinaridade é falar de disciplinas, de fronteiras e de conexões. Pensar a interdisciplinaridade na Economia é, em última análise, pensar a própria Economia como disciplina, o seu objecto, a natureza desse objecto, a definição das fronteiras e conexões que podemos ou devemos estabelecer. Neste texto dá-se conta da pluralidade de sentidos que a prática da interdisciplinaridade tem assumido na Economia, apresentam-se as razões por que se entende que a interdisciplinaridade é indispensável no estudo da economia e analisam-se dois modelos sobre o que deve constituir a interdisciplinaridade na Economia: um, multidisciplinar, o outro transdisciplinar. Chama-se ainda a atenção para os obstáculos inerentes à prática da interdisciplinaridade, desde os institucionais aos decorrentes das diferentes culturas disciplinares, modos e hábitos de pensar e conflitos intradisciplinares, defendendo-se, por fim, a relevância de uma concepção pluralista de ciência. 
O objectivo último do artigo é mostrar porque é que a economia (enquanto objecto de estudo), sendo um sistema aberto, não pode ser deixada apenas aos economistas.

PALAVRAS-CHAVE Interdisciplinaridade, Economia, isolações interdisciplinares, conexões, transdisciplinaridade, sistemas abertos, pluralismo.

\section{ABSTRACT}

This is a text on economics and interdisciplinary. It starts from the belief that thinking on interdisciplinarity is to reflect on the nature of disciplinarity itself, that talking of interdisciplinarity amounts to dealing with disciplines, boundaries and connections. Thinking on interdisciplinarity in economics is ultimately to reason on economics as a discipline, its subject, the nature of this subject and the definition of the boundaries and connections that we can or should establish. This text takes account of the plurality of meanings that the practice of interdisciplinarity has assumed in economics, presents the reasons why interdisciplinarity is considered to be essential to the study of the economy and analyzes two models of what should be interdisciplinarity in economics: one multidisciplinary, the other transdisciplinary. Reference is also made to the obstacles inherent in the practice of interdisciplinarity, from institutional barriers to those arising from different disciplinary cultures, habits of mind and ways of thinking and intradisciplinary conflicts; finally, the paper stands for the relevance of a pluralistic conception of science. The ultimate goal of this article is to show why the economy (as an object of study), being an open system, cannot be left just to economists.

KEYWORDS: Interdisciplinarity, Economics, interdisciplinary isolations, connections, transdisciplinarity, open systems, pluralism. 


\section{INTRODUÇÃO}

A interdisciplinaridade é hoje, como já foi observado ${ }^{1}$, uma palavra gasta, vulgarizada, quase vazia de sentido, frequentemente uma mera junção de perspectivas disciplinares distintas, sem substância nem resultados relevantes, quantas vezes pouco mais do que um "diálogo de surdos". Para alguns, pior do que isso, traduz simplesmente uma fuga às dificuldades, ao rigor e exigências da investigação especializada. Para muitos de nós representa um ideal a atingir, um projecto voluntarista que é preciso construir. Mas a interdisciplinaridade é também algo que "se está inexoravelmente a fazer, quer queiramos ou não". É, de facto, algo que "por nossa vontade e porventura independentemente dela, se vai fazendo" 2 . Pode defender-se, evidentemente, que discutir a questão da interdisciplinaridade não passa de um exercício diletante, inútil ou irrelevante para a prática concreta da investigação disciplinar. Não o creio. Como argumentei noutro lugar ${ }^{3}$, o modo como se pensa a interdisciplinaridade tem implicações importantes para o modo como a Economia é praticada (e também ensinada).

A interdisciplinaridade é uma palavra com múltiplos sentidos e pensável sob múltiplas perspectivas ${ }^{4}$. Para mim, pensar a interdisciplinaridade de forma consequente é, desde logo e inevitavelmente, pensar a natureza da própria disciplinaridade. Falar de interdisciplinaridade é falar de disciplinas, de fronteiras e de conexões. Pensar a interdisciplinaridade na Economia é pensar a própria Economia como disciplina, o seu objecto, a natureza desse objecto, a definição das fronteiras e conexões que podemos ou devemos estabelecer.

\footnotetext{
1 Pombo 2004a e 2004b.

2 Pombo 2004a: 20.

3 Neves 2012a.

${ }^{4}$ Arena et al. 2009; Kerstenetzky e Neves 2012; Pombo 2004b; Strober 2011.
} 
É, pois, de Economia que, em última instância, se falará neste texto. O objectivo é mostrar que, se é verdade que, como alguém já disse, "as fronteiras da mente e do hábito são mais difíceis de derrubar do que as fronteiras entre Estados", é também crucial compreender porque é que a economia ${ }^{5}$ não pode ser deixada apenas aos economistas. Neste sentido, começarei por fazer uma breve referência à evolução recente da Economia no que às suas fronteiras diz respeito, desde o chamado "imperialismo económico" até aos intensos processos de fertilização recíproca em curso (secção 2). A pluralidade de sentidos que a prática da interdisciplinaridade tem assumido na Economia deverá ficar clara neste contexto. Apresentarei de seguida (secção 3) as razões por que, em meu entender, a interdisciplinaridade é indispensável no estudo da economia. Na secção 4 analisar-se-ão dois modelos sobre o que deve constituir a interdisciplinaridade na Economia: um, enfatiza a necessidade de trocas com disciplinas "contíguas" tendo em vista um melhor conhecimento do sistema económico mantendo, contudo, a respectiva autonomia disciplinar (chamemos-lhe modelo multidisciplinar); o outro, mais radical, defende a necessidade de transcender as actuais disciplinas caminhando no sentido de uma ciência social unificada (chamemos-lhe modelo transdisciplinar). A secção 5 concluirá o texto. Chamar-se-á aí a atenção também para os obstáculos inerentes à prática da interdisciplinaridade. Desde logo os institucionais, mas também os decorrentes das diferentes culturas disciplinares e respectivos modos e hábitos de pensar e argumentar e as dificuldades associadas ao facto de as disciplinas serem, elas próprias, internamente conflituais. Neste contexto far-se-á breve menção à questão da interdisciplinaridade como interparadigmaticidade

\footnotetext{
5 Não havendo na língua portuguesa, como acontece na inglesa, duas palavras diferentes para distinguir a ciência económica (economics) da realidade que constitui o seu objecto de estudo (economy), adoptar-se-á, neste texto, a convenção de escrever Economia (com E maiúsculo) para referir a ciência e economia (com e minúsculo) quando está em causa a realidade que aquela ciência procura estudar.
} 
e sublinhar-se-á a relevância fundamental de um entendimento pluralista da ciência. Espera-se que, no fim, fique claro porque é que a economia não pode ser deixada apenas aos economistas.

\section{AS PRÁtICAS DA INTERDISCIPLINARIDADE NA ECONOMIA}

Qualquer disciplina, para se constituir, precisa definir o seu domínio, o seu centro de interesses e problemática teórica, e tal implica definir fronteiras face a outros discursos disciplinares. Implica "isolações interdisciplinares"6. Em resultado falamos, por exemplo, de discurso económico por contraste com os discursos da Física, da Biologia, da Psicologia, da Sociologia, da Ciência Política, da História, etc.

A constituição da Economia como ciência moderna, desde a publicação, em 1776, da famosa Riqueza das Nações ${ }^{7}$, por Adam Smith, até ao importante Essay on the Nature and Significance of Economic Science, de Lionel Robbins ${ }^{8}$, pode ser caracterizada como um longo processo de definição e autonomização de uma área de

\footnotetext{
6 Segundo Mäki 1992 e 2004, uma "isolação" ocorre quando algo, um conjunto de entidades $\mathbf{X}$ (o "campo isolado"), é subtraído à influência do restante universo, $\mathbf{Y}$ (o "campo excluído"). X, o campo isolado, e Y, o campo excluído, esgotam todas as possibilidades relevantes. As isolações podem ser teóricas ("thought experiments") ou materiais ("isolações experimentais" ou, mais raramente, "isolações espontâneas"). Falamos de "isolação teórica" à operação pela qual, na construção de um conceito, modelo ou teoria, um sistema, relação, processo ou característica é (intelectualmente) isolado de outras possíveis influências. As isolações interdisciplinares definem, assim, o domínio (e demarcam as fronteiras) de um dado discurso disciplinar face a outros domínios disciplinares. Existem, evidentemente, diferentes estratégias de isolação, as quais determinam o que é que é isolado (isto é, onde é definida a linha de fronteira entre $\mathrm{X}$ e $\mathrm{Y}$ ) e o modo como tal é realizado.

7 An Inquiry into the Nature and Causes of the Wealth of Nations.

8 Robbins 1932.
} 
estudos específica, com um objecto teórico bem delimitado e uma estrutura conceptual e método de investigação próprios.

É uma história muito rica e multifacetada, com múltiplos actores e variados contributos teóricos e metodológicos, que não cabe obviamente contar aqui. Refiram-se, ainda assim, a mero título de ilustração, alguns marcos cruciais deste processo, a saber:

i. A 'revolução marginalista' na década de 70 do séc. XIX - com a redefinição do "problema económico", deslocando o foco de atenção da esfera da produção e do estudo da origem da riqueza, do excedente económico e da questão da repartição (como acontecia na economia política clássica) para a esfera da troca e para o estudo do mercado e dos preços como mecanismo fundamental de coordenação económica e afectação de recursos - e a progressiva transformação, nos finais do séc. XIX/princípios do séc. XX, da Political Economy (Economia Política), tributária da Filosofia Moral, numa mais "científica" economics ("Economia") ;

ii. O destacado papel de Alfred Marshall, desde logo pela sua importante obra de consolidação teórica, mas também pelo seu papel na criação, em 1903, na Universidade de Cambridge (Reino Unido), de um plano de estudos de Economia (Economics and Politics Tripos) autónomo face ao tradicional Moral Sciences Tripos onde se inseriam anteriormente os estudos sobre a economia ${ }^{10}$;

iii. A famosa methodenstreit, uma longa controvérsia desencadeada pelo debate entre Carl Menger (escola austríaca) e Gustav von Schmoller (escola histórica alemã) opondo vigorosamente, entre as décadas de 80 do séc. XIX e a primeira do XX, duas

\footnotetext{
9 Vaggi e Groenewegen 2003, nomeadamente o cap. 17.

10 Groenewegen 2007.
} 
perspectivas polares acerca do estudo da economia - uma analítica, centrada na abstracção teórica (marginalismo), a outra enfatizando a necessidade de atenção aos factos históricos e aspectos institucionais ${ }^{11}$;

iv. Finalmente, mas não menos relevante, a obra metodológica de Lionel Robbins ${ }^{12}$, com a sua radical separação entre factos e valores (entre a ciência económica e a Ética), e o "consenso" ou "contrato implícito" ("gentlemen's agreement" nas palavras de Ingham) ${ }^{13}$ estabelecido entre economistas e sociólogos a partir de Talcott Parsons e Robbins em torno de uma divisão de trabalho centrada na perspectiva analítica de cada uma das disciplinas ("the analytical factor view"), focando-se cada uma delas num aspecto (factor) diferente da acção social - a Economia, "ciência da escolha", estudando a chamada escolha racional entre meios escassos para atingir fins dados (a acção "lógica", segundo Pareto); a Sociologia, "ciência da acção social", investigando o papel dos fins últimos e as atitudes e valores que lhes subjazem ${ }^{14}$.

Neste processo de definição e autonomização enquanto área de estudos específica, a Economia procura construir-se como uma ciência rigorosa, crescentemente matemática ${ }^{15}$, à imagem da Física - a mais "dura" das ciências "moles", como se dirá mais tarde - e adopta uma estratégia tendo em vista isolar analiticamente o estudo da esfera "económica" da influência das estruturas e relações sociais (separando-se,

\footnotetext{
11 Swedberg 1990; Hodgson 2001.

12 Robbins 1932.

13 Ingham 1996: 244.

14 Hodgson 2008; Velthuis 1999.

15 Mirowski 1991.
} 
desse modo, das restantes Ciências Sociais). Constitui-se, nas palavras de Ben Fine ${ }^{16}$, como uma "Economia Fortaleza".

Com Robbins a Economia torna-se, sobretudo, uma perspectiva de análise. O comportamento da economia é analisado em termos de soluções de equilíbrio (eficientes) para problemas de escolha racional de afectação de recursos escassos com utilizações alternativas (optimização), em resultado da agregação de comportamentos de agentes individuais considerados de forma atomista (modelo da escolha racional).

Mas o seu âmbito de estudo continuará a ser, até finais dos anos 50 do século $\mathrm{XX}$, o mercado e o mecanismo de preços (a par com os fenómenos monetários e ciclos económicos). A verdade, porém, é que nada na concepção de Economia de Robbins impunha tal limitação. Isso mesmo compreenderam vários autores, como Gary Becker e outros ${ }^{17}$, que, a partir da segunda metade dos anos 50, iniciam um percurso de transgressão da velha fronteira da Economia como ciência do mercado, assente na "exportação" do modelo da escolha racional para áreas tradicionalmente consideradas como "não-económicas" (escolhas educacionais, família, evolução demográfica, crime, discriminação, processos políticos, direito, etc.) ${ }^{18}$ e na aplicação dos princípios da optimização individual à análise das instituições e do comportamento colectivo. Para estes autores o social não é mais do que uma extensão do individual. O modelo da escolha racional pode e deve ser universalizado no quadro de uma nova "abordagem económica ao comportamento humano". Becker - porventura o mais notável e influente representante desta nova abordagem - diria a este propósito ${ }^{19}$ :

\footnotetext{
16 Fine 1997.

17 Como Anthony Downs, Gordon Tullok ou Mancur Olson.

18 Vejam-se, a título de exemplo, Becker 1976, Downs 1957, Posner 1972.

19 Becker 1976.
} 
"todo o comportamento humano pode ser visto como envolvendo participantes que maximizam a sua utilidade a partir de um conjunto estável de preferências e acumulam uma quantidade óptima de informações e outros inputs numa variedade de mercados."20

E, mais tarde, na sua Nobel Lecture ${ }^{21}$ :

"O modelo da escolha racional fornece a mais promissora base actualmente disponível para uma abordagem unificada à análise do mundo social por parte dos estudiosos das diferentes ciências sociais." 22

É, pois, de um “imperialismo da Economia" que se trata nesta tentativa de redefinição de fronteiras da Economia a que se assiste a partir dos anos 50 com base na exportação do método dominante na Economia para os outros domínios do social. ${ }^{23}$ A interdisciplinaridade não é aqui outra coisa senão um projecto de explicação de todo o comportamento social com recurso aos instrumentos da análise económica. Jack Hirshleifer, em The Expanding Domain of Economics ${ }^{24}$, fala mesmo da Economia como a "gramática universal" das ciências

20 "all human behavior can be viewed as involving participants who maximize their utility from a stable set of preferences and accumulate an optimal amount of information and other inputs in a variety of markets."

21 Becker 1993.

22 "The rational choice model provides the most promising basis presently available for a unified approach to the analysis of the social world by scholars from different social sciences."

${ }^{23}$ Uso aqui a designação que se tornou convencional na literatura, incluindo por defensores desta abordagem, como Edward Lazear (2000). Contudo, como notaram Olson e Kähkönen (apud Ben Fine 2002: 188), esta terminologia será inadequada já que não há recurso à força ou inibição da livre escolha. Aqueles dois autores sugerem, em alternativa, a sugestiva metáfora de uma Economia como metrópole estendendo a sua influência aos subúrbios das demais ciências sociais.

${ }^{24}$ Hirshleifer 1985. 
sociais considerando que as ciências sociais "não económicas" tenderão a tornar-se em breve indistinguíveis da ciência económica. Edward Lazear, outro entusiástico defensor do imperialismo económico, afirmou mais recentemente 25 : "O objectivo da teoria económica é unificar o pensamento e fornecer uma linguagem que possa ser usada para entender uma variedade de fenómenos sociais." 26

O imperialismo da Economia é ainda hoje um projecto com impacto no interior da disciplina e, sobretudo, sucesso mediático ${ }^{27}$. Na verdade, porém, a transgressão de fronteiras disciplinares a que temos assistido nas últimas décadas, ao contrário dos desejos redutores dos defensores do imperialismo económico, não tem obedecido a uma lógica estrita de exportação, devendo antes ser descrita como um movimento de duplo (ou até de múltiplos) sentido(s), um modelo de "exportação/ importação" 28 ou de "inspiração" mútua, ${ }^{29}$ caracterizado por intensos e diversos processos de contaminação conceptual e metodológica e fertilização recíproca.

Apenas alguns exemplos:

i. A aplicação da psicologia experimental e das neurociências ao estudo dos processos cognitivos e comportamentos na economia, da arquitectura da escolha e do design económico de mercados. 30

ii. A incorporação na Economia de abordagens assentes nas ciências da complexidade com aproximações entre a Física

25 Lazear 2000: 142.

26 "The goal of economic theory is to unify thought and to provide a language that can be used to understand a variety of social phenomena".

27 Veja-se, por exemplo, o sucesso da Freakonomics ("The Hidden Side of Everything"), http://freakonomics.com/.

28 Davis 2006.

29 Frey e Benz 2004.

30 Santos 2010 e 2012. 
e a Economia (e.g. teoria do caos e dinâmicas não lineares; economia computacional baseada em agentes). ${ }^{31}$

iii. A aplicação da teoria dos jogos aos estudos de conflito, cooperação e comportamento estratégico, por exemplo no domínio da Organização Industrial. 32

São profundas as mudanças que estas trocas interdisciplinares têm implicado nos pressupostos, conceitos e práticas da Economia. Conceitos-chave, como racionalidade e equilíbrio, e sua relevância têm vindo a ser significativamente repensados. As fronteiras com algumas disciplinas têm-se tornado bastante mais fluidas (é o que acontece, por exemplo, entre a Sociologia Económica e a abordagem institucionalista da Economia) ${ }^{33}$ levando mesmo, nalguns casos, ao desenvolvimento de novas áreas disciplinares e novas ou renovadas subdisciplinas. São exemplos particularmente salientes o crescimento acelerado da economia experimental e comportamental e da neuroeconomia (em resultado de um novo relacionamento com a Psicologia e as neurociências), a Economia da Complexidade, o desenvolvimento da "Law and Economics" (erradamente confundida, em meu entender, com uma redutora "análise económica do direito"), uma nova Economia Política Comparada (com os estudos sobre as variedades do capitalismo), ou ainda a chamada viragem normativa na Economia ${ }^{34}$ com o retorno à Ética.

Também na Economia se pode afirmar que muitos dos trabalhos mais inovadores têm estado associados a processos de fertilização recíproca nas fronteiras da disciplina, como alguns dos galardoados

\footnotetext{
${ }^{31}$ Fontana 2009; Arthur 2013; Graça e Lopes 2010.

32 van Damme 1999; Sugden 2001.

33 Ver, a este propósito, Dequech 2012.

${ }^{34}$ Kerstenetzky 2012.
} 
mais recentes com o Prémio das Ciências Económicas em Memória de Alfred Nobel ${ }^{35}$ bem ilustram.

Mas mais, há que referir que é ainda enorme o potencial de aplicação destes novos desenvolvimentos em variadíssimas áreas da Economia ${ }^{36}$. Esta vive actualmente um período de grande dinamismo não sendo particularmente arriscado prever que destes processos de fertilização interdisciplinar hão-de resultar no futuro próximo profundas transformações no estado actual da disciplina.

\section{PORQUE É A INTERDISCIPLINARIDADE IN DIS PENSÁVEL?}

As "isolações interdisciplinares" são inevitáveis. O conhecimento, como sublinhou Loasby ${ }^{37}$, cresce "por divisão". A relevância da divisão do trabalho de que falava Adam Smith na Riqueza das Nações é igualmente válida no que respeita ao conhecimento. As nossas limitações cognitivas (e as diferentes competências e skills que possuímos) impõem, sem dúvida, uma divisão do trabalho científico. O que podemos saber é necessariamente disperso. Mas é também incompleto e parcial. É bem conhecida a parábola dos cegos que, agarrados às suas percepções parcelares, são incapazes de identificar um elefante ${ }^{38}$. Por isso, como defende também Loasby ${ }^{39}$ :

"Uma sociedade rica em conhecimento deve ser uma ecologia de especialistas; o conhecimento é distribuído (...) e sendo distribuído

35 Mais conhecido, embora incorrectamente, como Prémio Nobel da Economia.

36 Diamond e Vartiainen 2007; Holt et al. 2011.

37 Loasby 1999: 135.

38 Ver, por exemplo, aqui: http://www.cs.princeton.edu/ rywang/berkeley/258/ parable.html e aqui: https://pt.wikipedia.org/wiki/Anekantavada.

39 Loasby 1999: 130. 
pode crescer, desde que seja suficientemente coordenado para suportar interdependências crescentes (ênfase minha).” 40

A perda de conexões relevantes - as interdependências de que fala Loasby na citação anterior - é efectivamente um problema da maior relevância. Conhecer é estabelecer conexões.

As isolações interdisciplinares implicam exclusões. Muitas conexões possíveis entre aspectos da realidade são descartadas, supostas inexistentes ou irrelevantes, a fim de prestar atenção a outras ${ }^{41}$. A escolha do "campo isolado" de uma ciência - que inclui o conjunto de itens explicativos (explanantia) e o conjunto de itens a explicar (explananda) - é também uma operação de exclusão. Tal acontece, em geral, por simples omissão. Os itens ou relações excluídos são ignorados e caem fora do âmbito da disciplina, sem qualquer menção. Tornam-se um "campo de silêncio" 42 .

Como aquilo que pode ser explicado por uma ciência é limitado pelo conjunto de itens explicativos escolhido 43 as isolações interdisciplinares envolvidas, quer na escolha do explananda, quer da explanantia - e as consequentes linhas de fronteira que daí resultam - são cruciais.

Várias estratégias de isolação interdisciplinar são observáveis no interior de cada disciplina - e a Economia não é obviamente excepção. Contrastem-se, por exemplo, as abordagens de Gary Becker e Ronald Coase. Becker alargou o âmbito de estudo da Economia incluindo nele todos os comportamentos humanos em face da escassez - escolhas educacionais, família, crime, etc. - procurando explicar

40 "Because different people can develop different skills, a knowledge-rich society must be an ecology of specialists; knowledge is distributed (...) and being distributed it can grow, provided that it is sufficiently co-ordinated to support increasing interdependencies".

${ }^{41}$ Loasby 2003: 294.

42 Mäki 1992: 335-6.

43 Maki 2004: 322. 
comportamentos até então considerados "não económicos" com recurso ao mesmo modelo da escolha racional que os economistas vinham há muito usando para analisar os comportamentos "económicos" de consumidores e produtores nos mercados. Ronald Coase, pelo contrário, apelou a um alargamento dos recursos explicativos dos economistas - incluindo neles qualquer contributo útil de outros campos disciplinares, nomeadamente do Direito - tendo em vista uma explicação mais adequada do objecto de estudo tradicional da Economia - o "sistema económico".

Controvérsias importantes têm, na verdade, ocorrido ao longo da história da Economia, tanto no que respeita à escolha dos itens a explicar como dos itens que se admite possam constituir factores explicativos. O que está em causa, muitas vezes, são disputas em torno de diferentes concepções sobre a natureza do objecto de estudo.

As fronteiras são, de facto, uma construção humana, mais ou menos artificial. O mundo não tem fronteiras, é ontologicamente uno. As isolações só existem no domínio do pensamento e das representações que fazemos acerca do mundo real; não nos fenómenos em $\mathrm{si}^{44}$. A economia que pretendemos estudar (o "sistema económico", segundo Coase) é um sistema aberto ${ }^{45}$.

O desafio, pois, é como adequar a teoria à natureza do objecto de estudo $^{46}$. Parece óbvia, depois do que se disse acima, a necessidade

\footnotetext{
${ }^{44}$ Loasby 1999: 14 .

45 Um sistema pode ser definido como um conjunto de elementos (coisas ou ideias) interligados entre si por uma rede de conexões formando um todo coerente - uma estrutura com conexões (Potts 2000). Um sistema pode ser fechado ou aberto. O primeiro é um sistema sem quaisquer relações com o meio envolvente ou com outros sistemas, um domínio circunscrito, não afectado por forças exteriores nem as afectando a elas. Em contrapartida, um sistema aberto é um sistema que, de algum modo, em maior ou menor grau, está conectado com o exterior (as fronteiras, a existirem, são fluidas e permeáveis). Uma excelente discussão, mais aprofundada, sobre a caracterização dos sistemas abertos pode ser encontrada em Chick e Dow 2005. Veja-se também Neves 2012b.

46 A prática científica pressupõe sempre uma ontologia subjacente (Ardebili 2005: 651). A nossa concepção sobre a realidade social - i.e., a nossa ontologia social - delimita o modo como teorizamos os objectos de estudo (ibid: 653): A lógica
} 
de decompor esse objecto. Mas como segmentar a realidade sem perder conexões fundamentais? Para além da restrição ontológica ${ }^{47}$ acima exposta - a nossa limitação cognitiva e a centralidade das interconexões do mundo real - precisamos de uma epistemologia da interdisciplinaridade.

É fundamental reconhecer, em primeiro lugar, o carácter provisório das fronteiras disciplinares. Como sustentam Arena et al. ${ }^{48}$ :

"Se a economia é ela própria holisticamente aberta, qualquer divisão do objecto de estudo em disciplinas será um tanto arbitrária, e também provisória. (...) O propósito dos modelos, teorias e sem dúvida disciplinas, é segmentar o objecto de estudo de modo a que não precisemos de considerar tudo de uma vez, ou apenas segundo uma única perspectiva, mas sim focando-nos em aspectos específicos do assunto em questão. Mas estas segmentações, para serem úteis num sentido holístico, têm de ser provisórias em vez de fixas. Assim, não somente os modelos são fechamentos provisórios enquanto auxiliares do pensamento, também as fronteiras disciplinares são elas próprias fechamentos provisórios que podemos querer mudar tendo em vista lidar com aspectos específicos da realidade económica.”49

de pensamento deve ser consistente [Ardebili diz "deve representar"] a lógica que assumimos existir na realidade social [a onto-logia] (ibid: 654).

47 Sobre o conceito de "restrição ontológica" e sobre a importância crucial de as nossas teorias respeitarem o que acreditamos ser o modo como o mundo funciona e sem o que não poderia funcionar como funciona ("the way the world works constraint") ver Mäki 2001.

48 Arena et al. 2009: 8.

49 "If the economy is itself holistically open, any division of the subject matter into disciplines is bound to be somewhat arbitrary, and also provisional. (...)The purpose of models, theories, and indeed disciplines, is to segment the subject matter so that we do not need to consider everything at once, or in only one way, but rather focus on particular aspects of the subject at hand. But these segmentations, to be useful in a bolistic sense, need to be provisional rather than fixed. Thus, not only are models provisional closures as aids to thought, but so disciplinary boundaries are themselves provisional closures which we may want to change for the purposes of addressing particular aspects of economic reality." 
Mas é igualmente basilar reconhecer que o conhecimento será sempre incompleto e parcial - haverá sempre conexões perdidas. A utopia da procura da totalidade e da unidade-na-diversidade irão inevitavelmente continuar connosco.

\section{MODELOS DE INTERDISCIPLINARIDADE NA ECONOMIA:} MULTIDISCIPLINARIDADE OU TRANSDISCIPLINARIDADE?

A questão mais difícil com que estamos confrontados quando pretendemos construir uma epistemologia da interdisciplinaridade é, como vimos antes, determinar o modo como a realidade objecto de estudo é segmentada tendo em vista potenciar as interconexões. $\mathrm{Ou}$, dito de outra forma: onde e como traçar linhas de fronteira?

Nesta secção focar-me-ei em dois grandes modelos sobre o que deve ser a interdisciplinaridade na Economia: um, que enfatiza a necessidade de trocas com disciplinas "contíguas" tendo em vista um melhor conhecimento do sistema económico mantendo cada uma das disciplinas a respectiva autonomia (chamemos-lhe modelo multidisciplinar); o outro, que defende a necessidade de transcender as actuais disciplinas caminhando no sentido de uma ciência social unificada (designemo-lo por modelo transdisciplinar).

Tomarei Ronald Coase como representante do primeiro modelo ${ }^{50}$. O modelo transdisciplinar foi extensamente desenvolvido por K. William Kapp ${ }^{51}$ mas propostas num sentido idêntico podem

\footnotetext{
50 Coase analisou a questão da interdisciplinaridade num pequeno texto, Economics and Contiguous Disciplines (Coase 1994 [1977]), e pronunciou-se sobre o tema das relações interdisciplinares da Economia em várias intervenções e entrevistas mais recentes (ISNIE Newsletter, vários números).

51 Kapp debruçou-se longamente sobre o tema da interdisciplinaridade em Toward a Science of Man in Society: A Positive Approach to the Integration of Social Knowledge (Kapp 1961), um livro ainda hoje fundamental, em meu entender, para a reflexão sobre este tema. Reflexões suas sobre a interdisciplinaridade podem
} 
ser encontradas em alguns autores contemporâneos como Geoffrey Hodgson e Tony Lawson ${ }^{52}$.

Para o modelo multidisciplinar a Economia, tal como as outras ciências sociais, é uma disciplina relativamente autónoma, com um objecto de estudo próprio - o "sistema económico", isto é, um conjunto de actividades, susceptíveis, em geral, de mensuração com base no padrão monetário ${ }^{53}$, dirigidas à produção, distribuição e uso de bens e serviços, e o quadro institucional em que se inserem ${ }^{54}$-, uma abordagem específica - enfoque na avaliação de custos e benefícios - e um adquirido de métodos/técnicas de investigação relativamente consolidado. Contudo, entende-se que o conhecimento do funcionamento do sistema económico exige que também a influência decisiva de factores ditos "extra-económicos" como os direitos de propriedade, o sistema educativo ou a actividade regulatória do Estado - estudados no âmbito de outras ciências sociais - seja tomada em devida consideração. O contributo de disciplinas "contíguas" é, pois, considerado da maior importância. A incursão dos economistas pelos territórios das outras ciências sociais, contrariamente ao que acontece no imperialismo económico, decorre assim, sobretudo, da necessidade de um melhor conhecimento do sistema económico. Sigamos o raciocínio de Coase ${ }^{55}$ :

ainda ser encontradas noutros textos, com destaque para os compilados em The Humanization of the Social Sciences (Kapp 1985).

52 Hodgson 1996 e Lawson 2003, cap. 6, em particular 161-164.

53 "The measuring rod of money". Como afirmou Alfred Marshall (Principles of Economics, $9^{\text {th }}$ variourum ed., apud Coase 1994 [1977]: 44): "the steadiest motive to ordinary business work is the desire for the pay which is the material reward of work. The pay may be on its way to be spent selfishly or unselfishly, for noble or base ends; ... but the motive is supplied by a definite amount of money: and it is this definite and exact money measurement of the steadiest motives in business life, which has enabled economics to outrun every other branch of the study of man."

${ }^{54}$ Empresas e outras estruturas organizacionais, mercados de bens e serviços, de trabalho e de capitais, sistema bancário, comércio internacional, etc.

55 Coase 1994 [1977]: 46, ênfase minha. 
"Os economistas podem (...) estudar outros sistemas sociais, como o jurídico e o político, não com o objectivo de oferecer um contributo para o direito ou a ciência política, mas porque tal é necessário para entender o funcionamento do próprio sistema económico. (...) partes desses outros sistemas sociais estão tão entrelaçados com o sistema económico que podem ser considerados tanto uma parte desse sistema como o são do sistema sociológico, político ou jurídico. (...) O estudo pelos economistas dos efeitos dos outros sistemas sociais sobre o sistema económico irá, creio eu, tornar-se, de forma permanente, uma parcela do trabalho dos economistas. Ele não pode ser feito eficazmente por cientistas sociais não familiarizados com o sistema económico. Esse trabalho pode ser realizado em colaboração com outros cientistas sociais mas é improvável que seja bem feito sem economistas. Por essa razão, penso que podemos esperar um alargamento permanente do âmbito da Economia de forma a incluir estudos em outras ciências sociais. Mas o objectivo será permitir-nos compreender melhor o funcionamento do sistema económico." 56

Ou seja: sem interdisciplinaridade não há conhecimento adequado do sistema económico, mas o contributo especializado dos economistas continua a ser imprescindível. O trabalho científico exige, nesta perspectiva, uma especialização que é ainda disciplinar.

\footnotetext{
56 "Economists may (...) study other social systems, such as the legal and political ones, not with the aim of contributing to law or political science but because it is necessary if they are to understand the working of the economic system itself. (...) parts of these other social systems are so intermeshed with the economic system as to be as much a part of that system as they are of a sociological, political or legal system. (...) The study by economists of the effects of the other social systems on the economic system will, I believe, become a permanent part of the work of economists. It cannot be done effectively by social scientists unfamiliar with the economic system. Such work may be carried out in collaboration with other social scientists but it is unlikely to be well done without economists. For this reason, I think we may expect the scope of economics to be permanently enlarged to include studies in other social systems. But the purpose will be to enable us to understand better the working of the economic system."
} 
Em contrapartida, para o modelo transdisciplinar, a necessária integração do conhecimento social exige que se transcendam as actuais disciplinas. O racional desta posição é que a "unidade essencial do objecto de estudo" das ciências sociais torna inadequada a especialização com base em disciplinas separadas e impõe que da presente compartimentação disciplinar se evolua para uma "ciência unificada [ou integrada] do homem em sociedade" (ou "ciência do homem e da cultura"57) com a substituição da actual especialização disciplinar por uma especialização temática ${ }^{58}$.

A ideia subjacente é que não existindo problemas puramente económicos ${ }^{59}$ não podem existir fundadas linhas de fronteira a separar a análise económica das outras áreas afins da investigação social. Se, argumenta Kapp ${ }^{60}$, se pretender ainda assim continuar a manter as linhas tradicionais de demarcação entre as ciências sociais especializadas, o resultado, quase inevitavelmente, será que:

"a perspectiva e o método preconcebidos vão determinar a selecção e definição dos problemas a serem investigados. Outros problemas que não podem ser tratados dessa maneira serão provavelmente ignorados ou empurrados para trás e para a frente na terra de ninguém entre as disciplinas. (...) Em vez de ser norteado pelo objecto de estudo, o especialista tende a investigar apenas os

57 Kapp 1985: 16.

58 Cfr. Kapp 1961: 201-6.

59 Algo que já sabemos pelo menos desde que, com a sua noção de "fenómeno social total", Marcel Mauss nos despertou para a incontornável unidade do objecto real das ciências sociais e para a inexistência de um sector da realidade social separável a que possamos chamar o "económico" (ver Nunes 1976: 13). A este respeito Kapp 1961: 201 escreveu: "[T]here are no purely economic or political problems in the real world. The unreal character of the problems which are traditionally defined as economic or political becomes evident as soon as we realize that we cannot distinguish, for instance, between economic and noneconomic satisfactions or between economic objectives and the search for power in international politics."

60 Kapp, 1961: 202. 
aspectos dos eventos sociais seleccionados a partir da perspectiva e com o auxílio da metodologia particular que a disciplina pré-seleccionou para ele como apropriadas." (ênfase minha) ${ }^{61}$

Ou seja: os problemas investigados pelas actuais ciências sociais especializadas são, segundo Kapp, parciais e porventura fictícios.

É o que acontece com a análise económica centrada na construção de um fictício bomo oeconomicus focado na escolha optimizadora (dita racional) entre recursos escassos para atingir fins exogenamente determinados e numa lógica de estrito cálculo "económico" ignorando qualquer outro tipo de considerações (éticas, políticas ou outras). Ao contrário, como argumenta Kapp, são necessários conceitos (ou estruturas conceptuais) integradores ("denominadores-comuns") que cruzem as várias disciplinas (e integrem tudo o que já sabemos a partir de cada uma delas) - como "homem"62, "cultura" ou "estrutura social" - e uma abordagem bolística capaz de tomar em devida conta todos os factores condicionantes do comportamento humano. Com efeito,

"Nenhuma teoria do comportamento humano que opere com determinantes únicos (seja a busca da riqueza, ou qualquer outra 'motivação') é susceptível de ser considerada adequada para a explicação do comportamento humano seja a que nível for e quaisquer que sejam as várias condições em que o homem age como consumidor, trabalhador, empresário, ou membro de um grupo social ou

61 "If the specialized disciplines nevertheless insist that the traditional lines of demarcation be maintained (...) [a]lmost inevitably the result will be that the preconceived perspective and method will determine the selection and definition of the problems to be investigated. Other problems which cannot be treated in this manner are likely to be ignored or shifted back and forth in the no-man's land between the disciplines. In short, instead of following the lead of the subject matter, the specialist (...) is inclined to investigate only selected aspects of social events from the perspective and with the aid of the particular methodology which the discipline has preselected for him as appropriate and proper."

62 Hoje diríamos "ser humano". 
político. Na verdade, qualquer tentativa de explicar a conduta humana em termos de 'motivações' ou determinantes únicos só pode produzir uma imagem simplista do comportamento humano ao destacar um factor de entre uma série de determinantes e atribuir-lhe potência causal." (ênfase minha) ${ }^{63,64}$

Além disso, defende Kapp, a lógica do cálculo económico assente na comparação de custos e benefícios com base nos preços do mercado - a possibilidade de recurso à "measuring rod of money" que parece ser a justificação para Coase de uma ciência económica autónoma - é questionável já que nem os custos de produção contabilizados pelas empresas representam os custos totais de produção (os custos efectivos de oportunidade) nem aqueles preços reflectem a verdadeira importância relativa das necessidades humanas. E, assim sendo, a valoração dos custos e benefícios sociais com base nos preços de mercado não fornecerá uma base sólida para a avaliação dos cursos desejáveis de acção a prosseguir.

A questão das fronteiras entre as várias ciências sociais será, assim, em última instância, uma questão de saber em que medida as abstracções e construções teóricas que elaboramos se adequam ou não à natureza do objecto de estudo ${ }^{65}$.

63 Kapp 1985: 18.

64 "No theory of human behavior which operates with single determinants (whether the pursuit of wealth, or any other 'drive') is likely to prove adequate for the explanation of human behavior at any level and under any of the various conditions under which man acts as a consumer, worker, entrepreneur, or member of a social or political group. In fact, any attempt to account for human conduct in terms of single drives or determinants can only yield an oversimplified picture of human behavior by singling out one factor from a number of determinants and attributing causal potency to it."

65 A este propósito, Kapp chama a atenção para a necessidade de as abstracções que fazemos serem derivadas a partir dos comportamentos observáveis - "tipos reais", definidos precisamente como "an abstraction derived from observed regularities in behavior", "an image that simplifies and renders intelligible what at first sight appears unconnected and disparate in character" - e não construções fictícias mais ou menos úteis. Para uma análise mais desenvolvida deste tópico ver Kapp 1961: 194-199. 
Para os defensores do modelo que estamos a analisar não há qualquer fundamento para uma ciência económica distinta e separável das outras ciências sociais ${ }^{66}$. A realidade (física, biológica e sociocultural) é estruturada em níveis cada vez mais elevados de organização e complexidade - matéria inanimada, organismos vivos e sociedade humana - os quais, embora conectados e interrelacionados entre si, se diferenciam por propriedades emergentes ${ }^{67} \mathrm{e}$ irredutíveis às do nível inferior. Cada nível de organização emergente, embora condicionado pelo(s) nível(eis) inferior(es), suscita novas questões e problemas que não podem ser adequadamente tratados com recurso aos conceitos e princípios utilizados para analisar o ou os outros níveis ${ }^{68}$. A realidade social não pode ser reduzida a explicações físicas ou biológicas. Uma ciência social, distinta das ciências da natureza, é necessária. Nada, porém, justificará a separação de problemas "económicos" relativamente ao resto da realidade social. Lawson coloca a questão do seguinte $\operatorname{modo}^{69}$ :

"Identificámos um domínio emergente de fenómenos especificamente económicos necessitando métodos relativamente distintos para a sua análise? Claro que não. E não é óbvio que tal seja possível. O mundo social, em todos os seus aspectos, gira à volta da prática humana, o primeiro explanandum da investigação social. E, quaisquer que sejam as práticas de interesse, entre o explanans das explicações sociais estão estruturas, posições, mecanismos, processos

\footnotetext{
66 Para além dos trabalhos de Kapp que temos vindo a seguir veja-se, no mesmo sentido, Lawson 2003: 164.

${ }^{67}$ Um nível ou estrato da realidade diz-se "emergente" ou com "propriedades emergentes" quando: (i) se desenvolve a partir de um nível "inferior", do qual depende para a sua existência e pelo qual é condicionado; mas que (ii) contém elementos e "poderes causais" próprios, irredutíveis aos que governam o estrato inferior e por isso não totalmente antecipáveis a partir destes, podendo eventualmente retroagir sobre as propriedades do nível inferior (Lawson, 2003: 44 e 161).

68 Kapp 1961: 75.

69 Lawson 2003: 162.
} 
e assim por diante. Por outras palavras, não há uma base óbvia para distinguir a Economia segundo a natureza do seu objecto, isto é, como uma ciência separada. E também não tem o seu próprio domínio. (...) A sua razão de ser não é um domínio separado de fenómenos distintos, com as suas próprias propriedades, mas um aspecto particular de toda a vida social." 70

\section{Kapp vai porventura mais longe quando escreve $\mathrm{e}^{71}$ :}

“Não há nenhum problema autónomo de crescimento económico e desenvolvimento. Assim que tentamos examinar porque é que alguns países são 'subdesenvolvidos' e outros não defrontamo-nos com círculos interconectados num processo de causalidade cumulativa em vez de factores puramente 'económicos' como escassez ou défice de recursos. Em suma, o chamado problema do desenvolvimento económico acaba por ser não um problema económico mas um problema sociocultural e político envolvendo mudanças estruturais de largo alcance." 72

70 "Have we identified an emergent realm of specifically economic phenomena, necessitating relatively distinct methods for their analysis? Clearly not. And it is not obvious that such is feasible. The social world, in all its aspects, turns upon human practice, the primary explanandum of social enquiry. And, whatever the practices of interest, amongst the explanans of social explanations are structures, positions, mechanisms, processes and the like. In other words, there is no obvious basis for distinguishing economics according to the nature of its object, i.e. as a separate science. Nor does it have its own domain. (...) Its raison d'être is not a separate domain of distinct phenomena with their own properties, but a particular aspect of all social life."

${ }^{71}$ Kapp 1961: 201-2.

72 "There is no autonomous problem of economic growth and development. As soon as we try to ascertain why some countries are 'underdeveloped' and others are not, we come upon interconnecting circles within a process of cumulative causation rather than purely 'economic' factors such as scarcities or shortages. In short, the so-called problem of economic development turns out to be not an economic problem but a socio-cultural and political problem involving far-reaching structural changes." 
No centro desta sua argumentação está a ideia de "causalidade cumulativa", ou de dinâmicas cumulativas, uma peça crucial na sua abordagem. São essas dinâmicas que fazem com que as alterações numa qualquer subestrutura do sistema social - parentesco e aculturação, produção e distribuição, sistemas políticos e sistemas noéticos de pensamento, crenças e valores - se traduzam em transformações do todo social no quadro de um processo aberto, circular e cumulativo de interacção e mudança ${ }^{73}$. Por isso, defende que em vez de uma estratégia (aditiva) de investigação das partes se deve colocar no centro da análise a própria interacção entre as partes e o todo - a rede de interconexões sociais - no contexto de uma abordagem "compreensiva" ou "holística" 74 . O processo dito "económico" deve ser entendido como um sistema inevitavelmente aberto, uma parte integrante de uma rede mais vasta de relações socioculturais.

Fica agora claro por que, no quadro deste modelo transdisciplinar, a necessária divisão do trabalho intelectual é deslocada da especialização disciplinar para uma especialização centrada em problemas ou áreas problemáticas determinada pela natureza dos mesmos ["Seguir os problemas até onde quer que eles nos possam levar"] 75 .

\section{NOTAS FINAIS}

Algumas ideias fundamentais podem ser consideradas adquiridas a partir da exposição que fizemos ao longo deste texto:

\footnotetext{
73 Kapp 1961, cap. VI.

${ }^{74}$ Kapp 1961: 180.

75 Kapp 1961: 205-6.
} 
i. Não há um objecto (real) distinto e separável - a economia - que possa ser considerado como património ou domínio dos economistas.

ii. Mas a divisão do trabalho intelectual é uma necessidade incontornável.

iii. As ciências definem-se não apenas pelo domínio da realidade que pretendem estudar ou pelo método que utilizam, mas pelo seu centro de interesses e pela problemática teórica que definem - que interrogações se colocam, que problemas pretendem resolver, em suma, qual o seu "objecto teórico". iv. Como defende Coase, o estudo da economia (como o de qualquer outra área de investigação) exige alguma familiarização com a natureza do objecto de estudo. Os economistas, na pluralidade de abordagens que tem caracterizado a disciplina ao longo dos últimos dois séculos e meio, têm já um largo património adquirido de conhecimentos sobre a realidade social que tomaram como seu foco de atenção privilegiado.

v. Contudo, a economia é demasiado complexa e interconectada com tudo o resto (desde os ecossistemas aos sistemas de valores e crenças) para poder ser acantonada no interior de uma disciplina. Sendo um sistema aberto, a economia pode ser segmentada em subsistemas susceptíveis de análise como se de sistemas fechados se tratasse, mas tais segmentações terão de ser sempre consideradas parciais e provisórias.

Os modelos de interdisciplinaridade que analisámos na secção anterior dão, como vimos, respostas substancialmente diferentes ao problema de saber como segmentar a realidade objecto de estudo tendo em vista potenciar as interconexões que sabemos existirem no mundo real (ou pelo menos minimizar a perda resultante da especialização disciplinar). Se a estratégia do modelo multidisciplinar é abrir as fronteiras disciplinares da Economia tendo em vista um 
melhor conhecimento do "sistema económico" preservando ainda assim a autonomia da disciplina, a estratégia que encontramos no modelo transdisciplinar, mais radical, aponta no sentido do abandono da especialização disciplinar substituindo-a por uma especialização temática. O racional destas duas estratégias terá ficado claro. O que está aí em causa terá ainda muito a ver com a resposta que damos à "velha" questão de saber o que privilegiar na definição da disciplina - o seu objecto de estudo ou a abordagem/método que perfilha.

Seja como for, e independentemente da posição que se possa assumir relativamente a esta questão, importa sublinhar que não há caminhos únicos para a prática da interdisciplinaridade. Como afirma Brian Loasby:

"Uma vez que as nossas representações são sempre incompletas, a inovação é sempre possível; podemos mudar o conjunto de elementos, rever as ligações internas entre eles ou redefinir as conexões externas. Quer contemplemos artefactos, processos, estruturas, sequências, problemas ou estratégias, estamos operando em grandes espaços combinatórios nos quais existem, em princípio, muitas opções de mudança." 76

Como defendi num outro texto ${ }^{77}$, a Economia é ela própria um sistema aberto e deve ser pensada enquanto tal.

Importa, para finalizar, chamar a atenção para o complexo problema dos escolhos que, em qualquer caso, uma abertura multi ou transdisciplinar suscita. De facto, são inúmeros os obstáculos à prática da interdisciplinaridade. Desde logo, obstáculos institucionais. A ciência, desde a investigação ao seu ensino, passando pelos critérios de reconhecimento - com impacto em termos de emprego

76 Loasby 2003.

77 Neves 2012b. 
e progressão nas carreiras de professores e investigadores - e pelo sistema de financiamento, assenta numa estrutura de base disciplinar e tende a reproduzir hábitos e práticas intrinsecamente disciplinares ${ }^{78}$. O sistema auto-reproduz-se e, de um modo geral, desincentiva a inovação multi/transdisciplinar. E mesmo quando a interdisciplinaridade é "algo que nós queremos fazer" 79 , somos frequentemente confrontados com dificuldades resultantes das diferentes culturas disciplinares e hábitos tão enraizados como os que têm a ver com modos de pensar e argumentar em cada disciplina, linguagens e critérios de avaliação acerca do que é ou não merecedor de atenção e susceptível de reconhecimento como tendo qualidade científica ${ }^{80}$.

Além disso, não se pode ignorar o facto de as disciplinas serem muitas vezes atravessadas por conflitos internos pelo que o diálogo interdisciplinar é, no âmbito das ciências sociais, não raras vezes, um esforço, sempre difícil, de cruzamento interparadigmático. Por isso, a discussão sobre a interdisciplinaridade tem de ser também uma discussão sobre o pluralismo no âmbito da ciência.

A interdisciplinaridade é um longo caminho a percorrer. Certo é - e julgo que isso terá ficado claro neste texto - que a economia, a realidade que tantos desde Adam Smith (e muitos ainda antes dele) procuram conhecer, é demasiado complexa para poder ser deixada apenas aos economistas.

78 Importa não esquecer que cada disciplina, sendo um empreendimento intelectual, é também uma estrutura institucional com uma história própria. A sua evolução depende não apenas de factores intelectuais mas também institucionais e técnicos (ao nível dos métodos de investigação) sendo fortemente condicionada pela sua história.

79 Pombo 2004a: 20.

${ }^{80}$ Vale a pena, a este propósito, ler dois textos fundamentais de Myra Strober sobre uma experiência de diálogo interdisciplinar levada a cabo em três universidades norte-americanas (Strober 2006 e 2011). 


\section{REFERÊNCIAS BIBLIOGRÁFICAS}

Ardebili, M. (2005), "Reorienting Economics, by Tony Lawson - Book Review", Review of Social Economy, 651-657.

Arena, R.; Dow, S.; Klaes, M. (2009), Open Economics: Economics in relation to other disciplines. Londres e Nova Iorque: Routledge.

Arthur, B. (2013), "Complexity Economics: A Different Framework for Economic Thought", Institute for New Economic Thinking (INET) Research Notes \#033 (disponível em http://ineteconomics.org/sites/inet.civicactions.net/files/Note-33-Arthur.pdf)

Becker, G. (1976), The Economic Approach to Human Behavior, Chicago: University of Chicago Press.

Becker, G. (1993), "Gary S. Becker - Prize Lecture: The Economic Way of Looking at Life". (disponível em http://www.nobelprize.org/nobel_prizes/economic-sciences/laureates/1992/becker-lecture.html)

Chick, V.; Dow, S. (2005), "The meaning of open systems", Journal of Economic Methodology, 12, 3: 363-381.

Coase, R. (1994 [1977]), "Economics and Contiguous Disciplines", in Coase, Ronald, Essays on Economics and Economists. Chicago: The University of Chicago Press, 34-46.

Davis, J. (2006), "The turn in economics: neoclassical dominance to mainstream pluralism?", Journal of Institutional Economics, 2, 1: 1-20.

Dequech, D. (2012), "Economia e Sociologia Econômica: Abordagens, Objetos e Práticas", in Kerstenetzky, Celia; Neves, Vítor (orgs.), Economia e interdisciplinaridade(s). Coimbra: Edições CES/Almedina, 149-172.

Diamond, P.; Vartiainen, Hannu (eds.) (2007), Behavioral Economics and its Applications. Princeton e Oxford: Princeton University Press.

Downs, A. (1957), An economic theory of democracy. Nova Iorque: Harper and Row.

Fine, B. (1997), "The new revolution in Economics", Capital \& Class 61: 143-148.

Fine, B. (2002), “Economic Imperialism': a view from the periphery”, Review of Radical Political Economics, 34: 187-201.

Fontana, M. (2009), "The Santa Fe Perspective on Economics: emerging patterns in the science of complexity", WP CESMEP Working paper No. 08/2009 (disponível em http://www.cesmep.unito.it/WP/2009/8_WP_Cesmep.pdf)

Frey, B. e Benz, M. (2004), "From imperialism to inspiration: a survey of Economics and psychology", in Davis, John et al. (eds.), The Elgar Companion to Economics and Philosophy. Cheltenham: Edward Elgar, 61-83.

Graça, J. C. e Lopes, . C. (2010), "Complexidade, interdependência e autonomia em sistemas económicos e sociais", Revista Crítica de Ciências Sociais [Online], 90 | 2010 (disponível em http://rccs.revues.org/1777)

Groenewegen, P. (2007), Alfred Marshall: Economist 1842-1924, Hampshire, UK e Nova York: Palgrave Macmillan. [tradução portuguesa: Alfred Marshall, Lisboa: Actual Editora, 2011]

Hirshleifer, J. (1985), "The Expanding Domain of Economics", The American Economic Review, 75, 6: 53-68. 
Hodgson, G. (1996), "Towards a Worthwhile Economics", in Medema, Steven; Samuels, Warren (eds.), How Economists Should Do Economics. Aldershot: Edward Elgar, 103-21.

Hodgson, G. (2001), How economics forgot history: the problem of historical specificity in social science. Londres e Nova Iorque: Routledge.

Hodgson, G. (2008), "Marshall, Schumpeter and the shifting boundaries of economics and sociology", in Shionoya, Yuichi; Nishizawa, Tamotsu (eds.), Marshall and Schumpeter on Evolution: Economic Sociology of Capitalist Development, Cheltenham, UK; Northampton, MA, USA: Edward Elgar.

Holt, R., Rosser Jr., B., Colander, D. (2011), "The Complexity Era in Economics", Review of Political Economy, 23, 3: 357-369.

Ingham, G. (1996), "Some recent changes in the relationship between economics and sociology", Cambridge Journal of Economics, 20: 243-275.

Kapp, K. W. (1961), Toward a Science of Man in Society: A Positive Approach to the Integration of Social Knowledge. The Hague: Martinus Nijhoff.

Kapp, K. W. (1976), "The Open-System Character of the Economy and its Implications", in Dopfer, Kurt (org.), Economics in the Future. Londres: The Macmillan Press, 90-105.

Kapp, K. W. (1985), The Humanization of the Social Sciences, organizado por Ullmann, John; Preiswerk, Roy, Lanham: University Press of America.

Kerstenetzky, C. (2012), "Economia e Ética: a virada normativa?", in Kerstenetzky, Celia; Neves, Vítor (orgs.), Economia e interdisciplinaridade(s). Coimbra: Edições CES/Almedina, 55-76.

Kerstenetzky, C.; Neves, V. (orgs.) (2012), Economia e Interdisciplinaridade(s). Coimbra: Almedina.

Lawson, T. (2003), Reorienting economics. Londres e Nova Iorque: Routledge.

Lazear, E. (2000), "Economic Imperialism", The Quarterly Journal of Economics, 115, 1, 99-146.

Loasby, B. (1999), Knowledge, Institutions and Evolution in Economics, Londres e Nova Iorque: Routledge.

Loasby, B. (2003), "Closed models and open systems", Journal of Economic Methodology, 10, 3: 285-306.

Mäki, U. (1992), "On the Method of Isolation in Economics", Poznan Studies in the Philosophy of the Sciences and the Humanities, 26, 317-351.

Mäki, U. (2001), "The way the world works (www): towards an ontology of theory choice", in Mäki, Uskali (ed.), The Economic World View: Studies in the Ontology of Economics, Cambridge: Cambridge University Press, 369-389.

Mäki, U. (2004), "Theoretical isolation and explanatory progress: transaction cost economics and the dynamics of dispute", Cambridge Journal of Economics, 28, 319-346.

Mirowski, P. (1991), "The When, the How and the Why of Mathematical Expression in the History of Economics Analysis", The Journal of Economic Perspectives, 5, 1: 145-157.

Neves, V. (2012a), "A análise dos custos sociais em Ronald Coase e K. William Kapp: duas perspectivas sobre a economia e a interdisciplinaridade", in Kerstenetzky, 
Celia; Neves, Vítor (orgs.), Economia e interdisciplinaridade(s). Coimbra: Edições CES/Almedina, 125-147.

Neves, V. (2012b), "Sciences as Open Systems - The Case of Economics", in Pombo et al. (eds.), Special Sciences and the Unity of Science. Dordrecht: Springer, 205-217.

Nunes, A. S. (1976 4 ed.), Questões Preliminares sobre as Ciências Sociais. Lisboa: Gabinete de Investigações Sociais.

Pombo, O. (2004a), Interdisciplinaridade: Ambições e Limites. Lisboa: Relógio d'Água.

Pombo, O. (2004b), "Epistemologia da interdisciplinaridade", in Pimenta, Carlos (Org.), Interdisciplinaridade, Humanismo, Universidade. Porto: Campo das Letras, 93-124.

Posner, R. (1972 1st edition), Economic Analysis of Law. Boston: Little, Brown and Company.

Potts, J. (2000), The New Evolutionary Microeconomics: Complexity, Competence and Adaptive Behaviour. Cheltenham: Edward Elgar.

Santos, A. (2010), "Terá chegado o tempo dos arquitectos e designers económicos?", in Neves, Vítor; Caldas, José, A Economia Sem Muros. Coimbra: Edições CES/ Almedina, 117-138.

Santos, A. (2012), "Entre a Economia e a Psicologia: comportamento e experiências", in Kerstenetzky, Celia; Neves, Vítor (orgs.), Economia e interdisciplinaridade(s). Coimbra: Edições CES/Almedina, 173-196.

Strober, M. (2006), "Habits of the Mind: Challenges for Multidisciplinary Engagement", Social Epistemology: A Journal of Knowledge, Culture and Policy, 20, 3-4, 315-331.

Strober, M. (2011), Interdisciplinary conversations: challenging habits of thought. Stanford, CA: Stanford University Press.

Sugden, R. (2001), "The evolutionary turn in game theory", Journal of Economic Methodology, 8, 1: 113-130.

Swedberg, R. (1990), "The New 'Battle of Methods', Challenge, 33, 1: 33-38.

Vaggi, G. e Groenewegen, P. (2003), A Concise History of Economic Thought From Mercantilism to Monetarism. Hampshire, UK e Nova York: Palgrave Macmillan.

van Damme, E. (1999), "Game Theory: the Next Stage," in Kirman, A.; Gérard-Varet, L-A. (eds.), Economics Beyond the Millennium. Oxford: Oxford University Press, 184-214.

Velthuis, O. (1999), "The Changing Relationship Between Economic Sociology and Institutional Economics: From Talcot Parsons to Mark Granovetter", American Journal of Economics and Sociology, 58, 4: 629-649. 
OBRA PUBLICADA

COMA COORDENAÇÃO

CIENTÍFICA

$\mathrm{ECH}$

- U 